\title{
le sourire en prothèse ou l'éloge du sourire
}

RÉSUMÉ Le sourire est bien sûr un réflexe naturel, mais s'y ajoute son rôle dans l'expression d'un visage, d'un sentiment, d'une attirance. Cela rend cet acte complexe tant au niveau de sa compréhension que de sa reproduction.

Ces facteurs imposent aux praticiens :

- d'une part d'analyser les différentes composantes selon une approche précise : analyse du cadre facial, labial, dentaire ;

- d'autre part de reproduire avec les techniques et procédés actuels, ces différentes composantes en particulier en

\section{Olivier HUE}

Chirurgien-dentiste,

Faculté de chirurgie dentaire Garancière,

5 , rue Garancière,

75006 Paris. individualisant chaque dent par sa position, sa forme et sa teinte.

\section{MOTS CLÉS}

sourire

plan d'occlusion

teinte

couleur

esthétique 


\section{introduction}

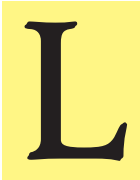

e sourire est un réflexe naturel associant les lèvres et les yeux, réflexe qui traduit toutes nos émotions. Ne parle-t-on pas de sourire rêveur, absent, mesquin, sarcastique, admiratif, ironique etc. Les adjectifs susceptibles d'être accolés au mot sourire s'avèrent innombrables[1].

Mais si, en dehors de la poésie, le sourire fut très longtemps ignoré par l'art, il est de nos jours au centre de toutes nos préoccupations. Aucune photo de magazine, de publicité ne saurait être publiée sans que la personne photographiée, actrice de sa propre vie ne souriât.

Face à cette réalité, il incombe au praticien de comprendre, de découvrir, si elles existent, les règles du sourire afin de les rétablir ou de les restaurer à l'aide des protocoles et techniques prothétiques et autres mis à notre disposition.

\section{le sourire : pourquoi son attirance?}

Il est éminemment paradoxal que le sourire d'un invidu attire tant notre attention. En effet, si l'on se réfère aux proportions académiques $\mathrm{du}$ corps humain le visage ne représente que $12 \%$ de la hauteur corporelle et les lèvres, actrices majeures du sourire, $2 \%$ (fig. 1).
Mais en réalité le sourire est un moyen de communication exceptionnel par sa richesse, sa complexité, sa diversité.

Moyen d'expression : le jeu plus ou moins ample, plus ou moins complexe des différents muscles de la mimique permet d'exprimer des joies, de l'inquiétude, de l'angoisse.

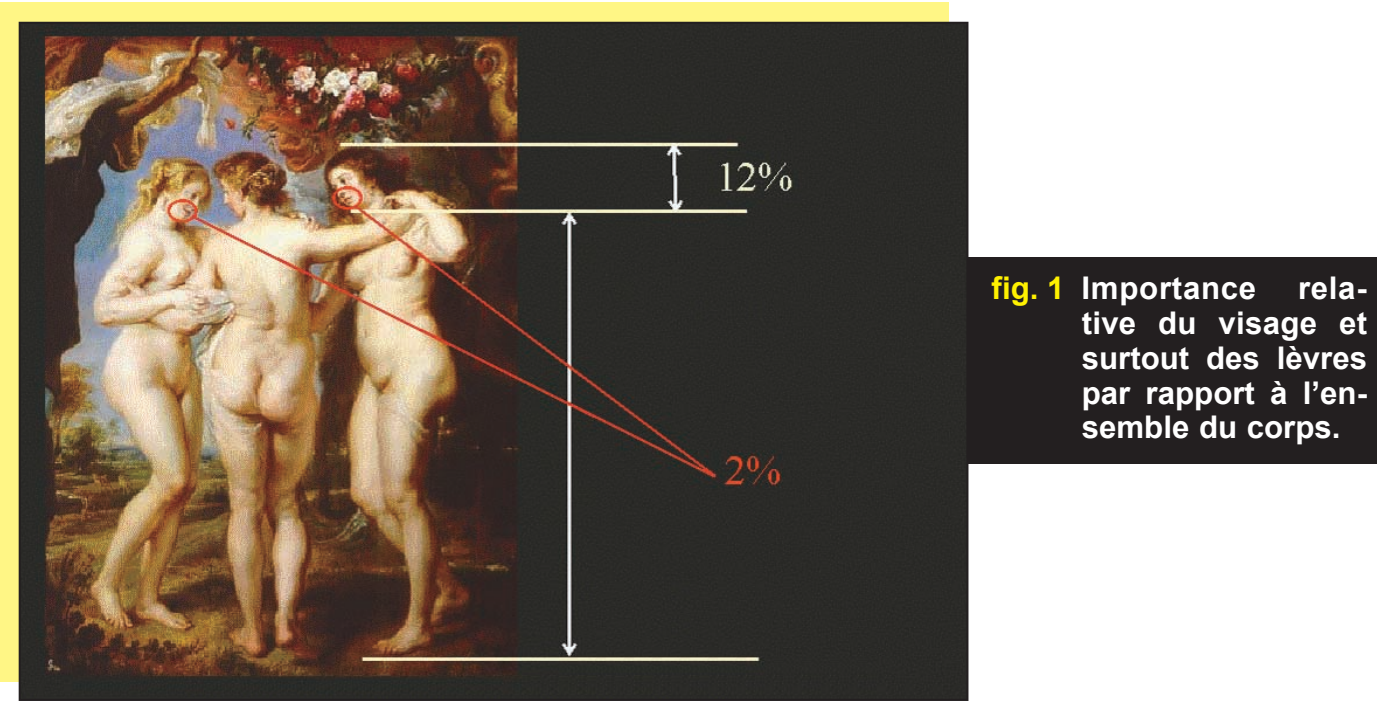


Ermiane, en son temps, en fit une description précise, en classant ces muscles en muscles de la joie, de la tristesse, de l'autorité, de l'efficacité[2].

Moyen d'affirmation : dans notre civilisation occidentale actuelle, le sou- rire contribue de manière importante à l'affirmation de la personnalité. Pour preuve, la multitude de photographies qui font appel à des personnes de tout âge, bronzées, souriant largement à dents déployées, blanches, bien alignées etc.

\section{le sourire : comment l'analyser ?}

Au niveau du visage, le sourire, composant essentiel de l'esthétique en général et du visage en particulier doit répondre aux critères définis par Aristote: "Le Beau nécessite précision, symétrie, coordination".

L'analyse $d u$ visage porte successivement sur : le cadre facial, le cadre labial, le cadre dentaire ${ }^{[3-4]}$ (fig. 2).

\section{- le cadre facial}

Dans le plan frontal, les critères précédents sont définis par les trois plans suivants :
- le plan sagittal médian : ce plan passe par le milieu du visage définissant ainsi un plan de symétrie. Cependant, ce dernier met en évidence un des paramètres essentiels de l'esthétique : l'absence de symétrie réelle du visage. Cette dissymétrie «naturelle», devra, cependant, toujours demeurer «harmonieuse» ;

- le plan bipupillaire : passant par les pupilles des deux yeux, il définit «l'horizontalité» du visage ;

- le plan bicommissural : il passe par les commissures labiales, il devrait être parallèle à la ligne bipupillaire, et ces

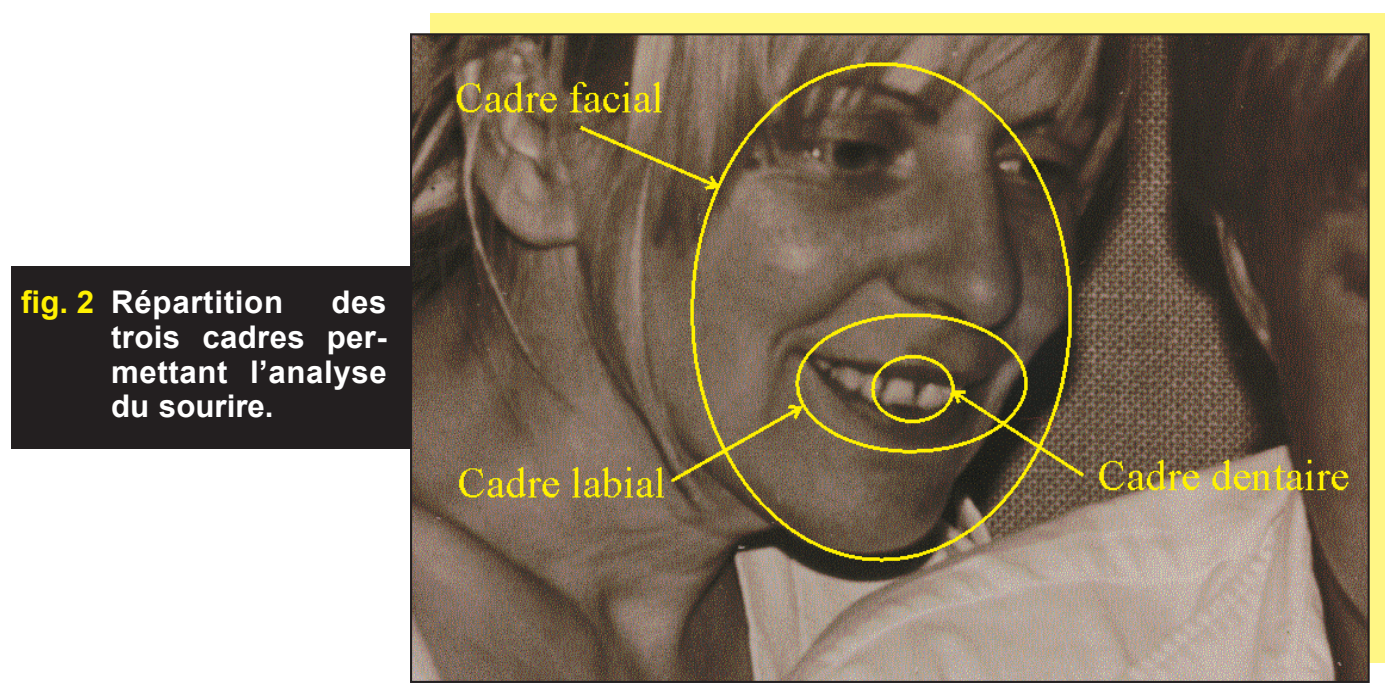



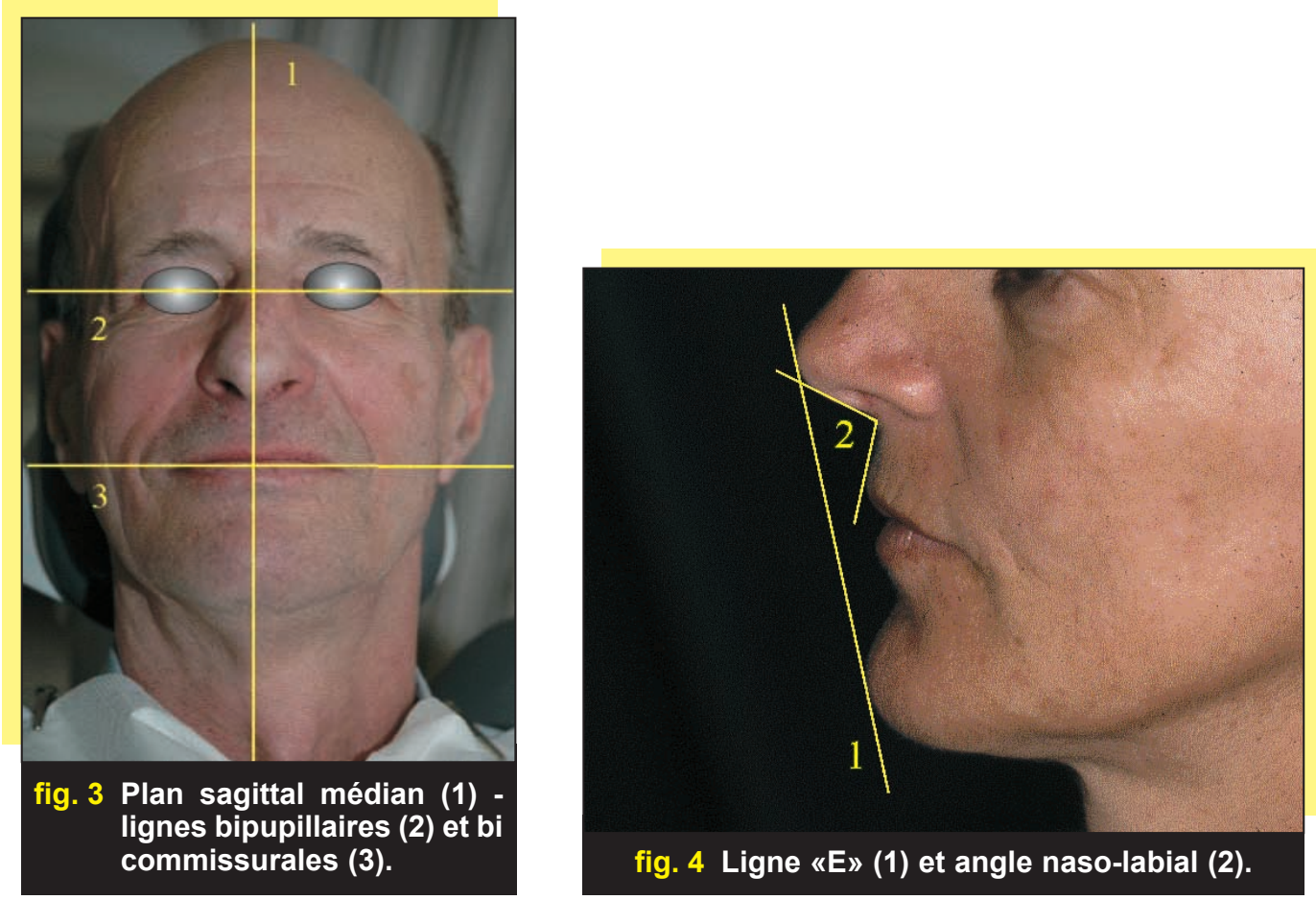

deux derniers plans perpendiculaires au plan sagittal médian (fig. 3).

Dans le plan sagittal, l'analyse du volume et de la position des lèvres fait appel aux deux critères suivants :

Angle naso-labial : il est formé par l'intersection entre deux tangentes, l'une au niveau de la base du nez, l'autre au bord externe de la lèvre supérieure. Normalement, cet angle varie entre $90^{\circ}$ et $95^{\circ} \mathrm{chez} l^{\prime}$ homme et $100^{\circ}$ à $105^{\circ}$ chez la femme. Son ouverture est souvent provoquée par une perte des dents antérieures[5].

Ligne E ou ligne de Ricketts : tangente entre la pointe $d u$ menton et $d u$ nez, elle permet d'évaluer la position des lèvres. Normalement, la lèvre supérieure se situe $4 \mathrm{~mm}$ en arrière et la lèvre inférieure $2 \mathrm{~mm}$. Cependant ces

valeurs sont soumises à des variations significatives d'origine sexuelle, raciale (fig. 4).

\section{- le cadre labial}

Le cadre labial est un cadre à la fois statique et dynamique. Au repos, l'incontinence labiale masque les unités dentaires, alors que lors du sourire celles-ci apparaissent, mettant en exergue l'organisation des dents.

\section{ASPECT STATIQUE}

L'harmonie des lèvres, critère d'une esthétique idéale, est basée sur les paramètres morphopsychologiques participant à l'équilibre du visage qui dépendent des éléments suivants : 


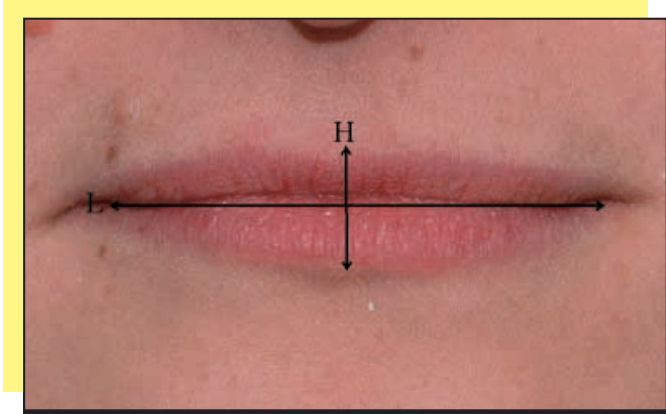

fig. 5 Dimension des lèvres $4 \mathrm{H}=\mathrm{L}$.

Dimensions : selon un rapport géométrique, idéalement, la largeur des lèvres est égale à 4 fois la hauteur. Bien sûr, il existe de nombreuses variations, mais ces critères permettent de définir plus précisément les caractéristiques de la lèvre: la brièveté par exemple (fig. 5).

Philtrum : cette fossette située dans le plan sagittal médian est un élément essentiel de l'harmonie de la lèvre supérieure. Son nom latin est issu du grec ancien $\varphi^{i} \lambda \tau \rho o v$ «moyen de se faire aimer» et, par extension, «fossette au-dessus de la lèvre supérieure», venant à son tour de $\varphi \imath \lambda \varepsilon ́ \omega$ «aimer par amitié». De par son étymologie, on peut comprendre tout son rôle dans l'analyse et l'appréciation d'un sourire[7].

Commissures : en dehors du plan qu'elles créent, elles correspondent à l'union des lèvres supérieure et inférieure. Chaque commissure est bordée par une petite éminence cutanée sur laquelle se termine le sillon nasogénien. Elles sont situées, idéalement, au repos à l'aplomb de la pupille correspondante lorsque l'œil regarde en avant vers l'infini.

\section{ASPECT DYNAMIQUE}

Lors du sourire, les lèvres se soulèvent de manière plus ou moins importante, laissant apparaître les arcades dentaires qui donnent une autre dimension à l'expression $d u$ visage, dimension qui peut être favorable ou défavorable en fonction de l'état de la denture.

Les composants du cadre labial dynamique sont au nombre de cinq :

\section{Exposition des dents au repos:} au repos, l'incontinence labiale laisse apparaître les bords libres des dents antérieures. Le degré de visibilité est fonction de l'âge, du sexe, de la longueur de la lèvre, du type racial[8].

- Âge: chez les sujets jeunes les dents maxillaires sont les plus visibles, mais vers $30-40$ ans, la visibilité des dents supérieures s'estompe au profit des dents mandibulaires ; situation qui est, esthétiquement, unanimement rejetée.

- Sexe : chez les femmes la visibilité des dents, $3,40 \mathrm{~mm}$ pour les incisives maxillaires et $0,5 \mathrm{~mm}$ pour les incisives mandibulaires, est deux fois plus importante que chez les hommes (1,91 mm).

- Longueur de la lèvre : elle est en relation directe avec le degré d'exposition des dents antérieures. Lorsque la lèvre supérieure est courte les dents supérieures sont très visibles et les inférieures peu, alors que lorsque la lèvre supérieure est très longue le degré de visibilité des dents s'inverse.

- Race : l'exposition des dents antérieures est constante chez les noirs et les asiatiques alors que chez les populations caucasiennes seules les dents maxillaires sont majoritairement exposées. 


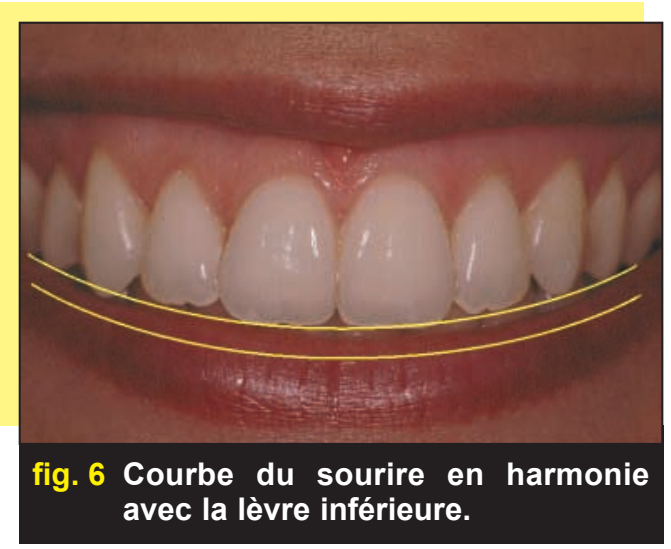

Courbe du sourire : c'est un élément essentiel de l'attirance indéfinissable qu'exerce le sourire. Elle correspond à une ligne courbe selon laquelle les bords libres des dents antéro-supérieures épousent la lèvre inférieure. Cette harmonie idéale s'installe dans $75 \%$ des cas, mais deux autres situations cliniques se présentent[9] (fig. 6).

Limite du sourire : lors du sourire, la lèvre supérieure découvre plus ou moins les faces vestibulaires des dents. La limite $d u$ découvrement peut être basse ne laissant que très peu apparaître les structures dentaires, moyenne les papilles interdentaires apparaissent très légèrement, haute mettant en évidence les structures gingivales.

Corridor labial : le corridor labial est l'espace qui apparaît lors du sourire entre les faces vestibulaires des dents maxillaires et la face interne des joues. Cet espace contribue à la création d'une perspective à la fois par la création d'une ligne de «fuite» et par la diminution de la luminosité des dents.

Bord libre : la position frontale et sagittale des bords libres vis-à-vis de la lèvre inférieure est capitale. Dans l'idéal, lors du sourire ils doivent affleurer celle-ci voire se situer à légère distance. Par contre il est peu souhaitable que les bords libres soient recouverts par la lèvre inférieure.

Plan d'occlusion ou plan incisif : celui-ci correspond à la partie antérieure du plan occlusal dans le plan frontal ; il est formé par les bords libres des incisives voire des canines maxillaires. Il doit être parallèle aux lignes bipupillaire et bi-commissurale et donc perpendiculaire au plan sagittal médian.

\section{cadre dentaire}

C'est un cadre, certes plus restreint, mais dans lequel les principes, les lois, les paramètres de l'esthétique sont aussi parfaitement définis. Ceux-ci doivent être analysés, respectés, appliqués quel que soit le tropisme ou la sensibilité du praticien.

\section{SYMÉTRIE}

La notion de symétrie est essentielle dans l'organisation de l'arcade dentaire. Elle doit s'analyser selon le principe de la symétrie horizontale et de la symétrie radiante[10].

- Symétrie horizontale: il s'agit d'une image identique mais inversée par rapport à un plan dit «plan de symétrie».

- Symétrie radiante: la symétrie s'installe à partir d'un point central, au niveau du sourire, le plus souvent par rapport au point de contact inter-proximal (fig. 7).

Cette notion de symétrie radiante et horizontale se retrouve dans l'organisation des contours gingivaux, limites cer- 


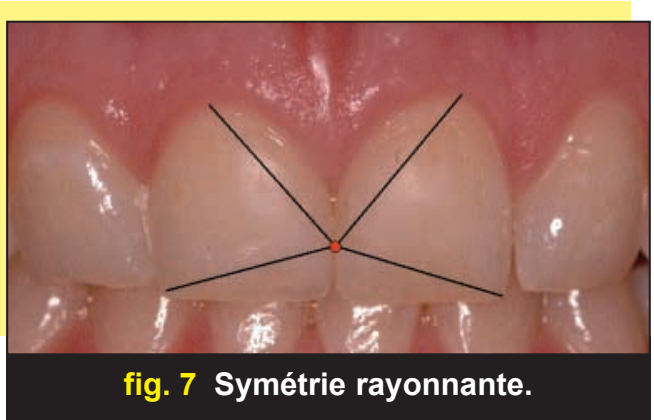

vicales, hauteur et position des limites cervicales, formes des papilles inter proximales en harmonie avec les lignes des points de contact interproximaux.

\section{PROPORTION}

La notion de proportion est un des principes fondamentaux de l'esthétique. Il en est pour preuve la multiplicité des canons de la beauté qui ont été développés au cours des siècles. Ces proportions sont basées sur des observations naturelles. Les feuilles, les fleurs, les coquillages se développent avec des proportions considérées comme idéales. Leurs croissances sont étroitement reliées à une progression mathématique dite série de Fibonacci (Mathématicien italien 1170-1205). Dans cette série, un nombre est égal à la somme des deux précédents $(1,1,2,3,5,8,13,21,44$ etc.). Le rapport entre les nombres de cette série s'approche d'une valeur de 1,618 chiffre mieux connu sous le terme de nombre d'Or[11].

L'utilisation de ce rapport a été particulièrement mise en exergue au niveau de l'agencement des dents antérieures en particulier des dimensions mésio-distales des incisives et canines maxillaires (fig. 8). Des compas, des abaques, ont été développés pour exploiter ce

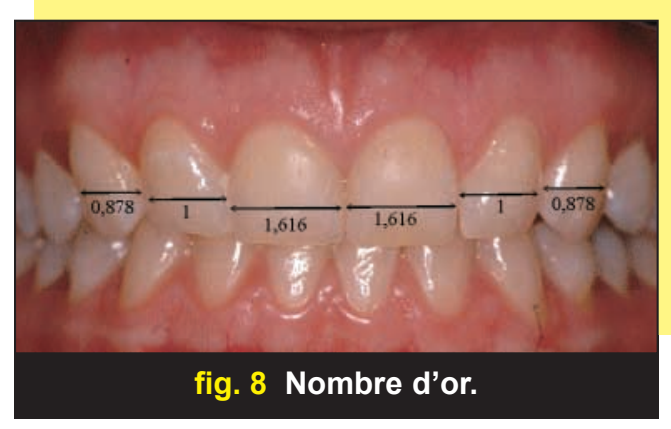

rapport. Cependant, celui-ci n'existe que très peu dans la nature : $17 \%$ selon Preston[12]. De plus, il semblerait que le respect de cette proportion divine, se traduirait pour le praticien et les patients par un aspect esthétique moins agréable.

\section{FORME}

Depuis Léon Williams, il est habituel de donner aux dents des formes générales : carrées, triangulaires, rondes, carrées à modification ovoïde. Cependant, une notion essentielle demeure celle de leurs proportions. Pour une incisive centrale, les proportions s'établissent aux environs de $80 \%$, rapport idéal pour Brisman, mais pour Chiche et Pinault ce rapport se rapproche plus de $75 \%[13-14]$.

\section{COULEUR}

La couleur est une composante de l'esthétique extrêmement complexe. Dans une première analyse, la couleur se compose de trois paramètres majeurs : luminosité, saturation, teinte. Mais à celles-ci s'ajoutent diverses caractérisations : externes majeures, reliefs plus ou moins marqués de la surface, de l'état de surface ou internes, présence 
de lignes verticales brunes, opalescentes, de tâches brunes, claires etc.

\section{ÉTAT DE SURFACE}

L'état de surface des dents naturelles est un élément essentiel de leur apparence, de leur visibilité.

En effet, les surfaces dentaires sont marquées par des macro reliefs naturels tels des lobes, des concavités, des convexités et par des microreliefs. Ces derniers sont formés de minuscules rai- nures le plus souvent horizontales. L'ensemble de ces reliefs répartit différemment la réflexion et la diffusion de la lumière incidente sur les surfaces dentaires modifiant de manière importante leur visibilité.

Lorsque la lumière est simplement réfléchie, la visibilité de la dent s'accroît, lorsque la diffusion augmente la visibilité diminue. De même, les concavités créent des ombres foncées donc de la profondeur, alors que les convexités créent de la lumière et du relief.

\section{le sourire : comment le rétablir en prothèse ?}

L'ensemble des techniques et procédés prothétiques actuels permettent de répondre de manière extrêmement positive à la majorité des situations cliniques.

Celles-ci autorisent des restaurations prothétiques moins invasives,
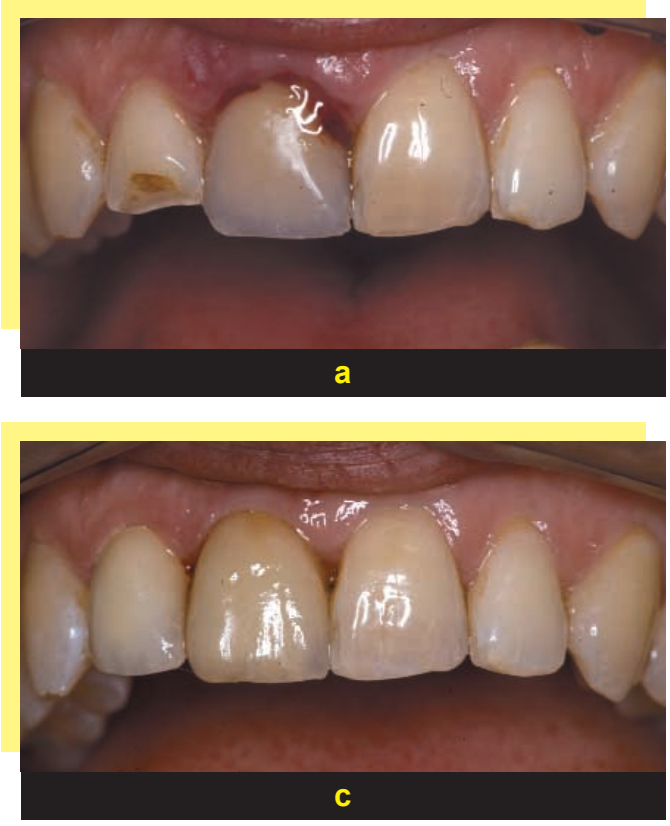

plus biologiques, plus esthétiques que ce soit par l'apport des bridges collés, des restaurations unitaires ou plurales sur implants, des céramo-céramiques (fig. 9 a à c).

Reste le rétablissement $d u$ sourire grâce au respect des principes et règles

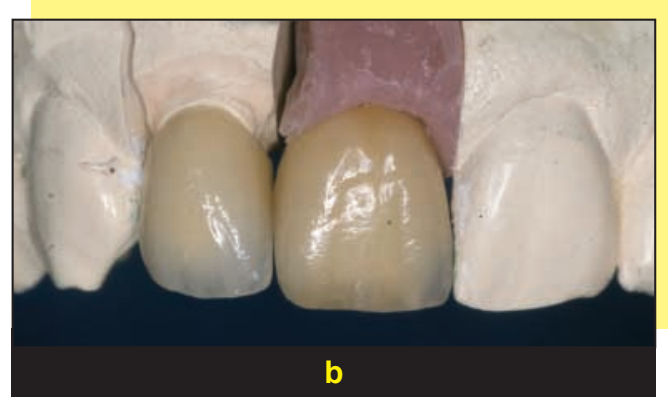

\section{fig. 9 a à $\mathrm{c}$}

a: fracture radiculaire de l'incisive centrale et coronaire de l'incisive latérale ;

b: couronne céramométallique sur implant au niveau de l'incisive centrale et céramocéramique au niveau de la latérale ;

c : résultat esthétique. 
qui s'y rattachent et qui s'adressent à tous les âges.

\section{- prothèse chez l'enfant}

Les agénésies partielle ou totale, quelle qu'en soit l'origine, provoquent nombre de problèmes chez les enfants, en particulier psychologiques par absence d'un beau sourire. La réalisation de prothèses amovibles chez ces patients contribue grandement à rétablir leur confiance et leur insertion dans leur monde (fig. $10 \mathrm{a}$ à c).

\section{- prothèse chez l'adulte}

A ce stade, les solutions prothétiques sont bien évidemment, plus nom-
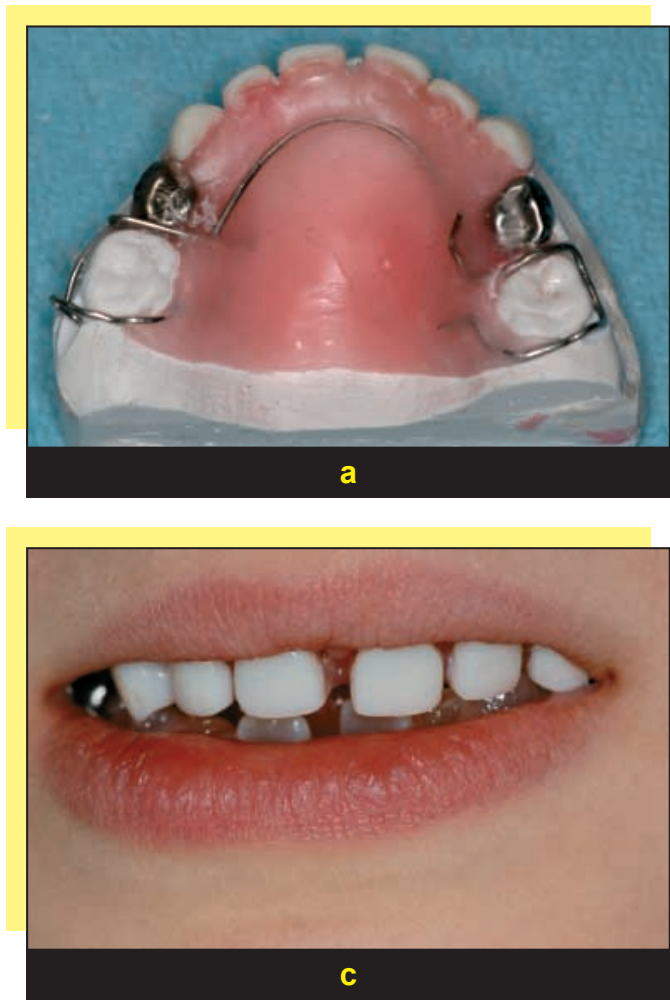

breuses, plus complexes tant par la difficulté des situations cliniques que par les souhaits, espérances, exigences esthétiques des patients, autant de problèmes auxquels les praticiens se trouvent confrontés.

Mais, une question se pose: doit-on appliquer de manière stricte les principes esthétiques d'Aristote ? Goldstein répond oui ! mais en précisant qu'il convient d'individualiser les dents par leur position, leur forme, leur teinte[15].

\section{INDIVIDUALISATION PAR LA POSITION}

La position des dents est déterminée entre autre par le principe de symétrie :

Symétrie réelle: géométriquement, la symétrie absolue existe. Par contre chez l'être vivant, en particulier chez les primates, elle n'existe pas.

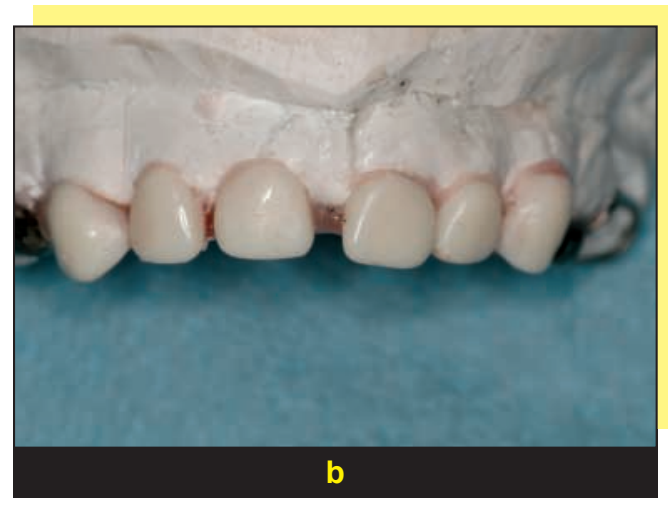

\section{fig. $10 \mathrm{a}$ à $\mathrm{c}$}

a : prothèse adjointe partielle pédiatrique vue occlusale ;

b : prothèse adjointe partielle pédiatrique vue vestibulaire ;

c : résultat final. 
Plan de symétrie : le choix du milieu d'un visage donc du plan de symétrie est délicat. Plusieurs repères ont été proposés : la papille rétro-incisive, le frein de la lèvre, la gouttière philtrale, le milieu du visage. Ces deux derniers repères semblent les plus fiables. De plus, le milieu du visage et milieu inter-incisif ne sont alignés que dans $30 \%$ des cas.

Symétrie des dimensions : toutes les études démontrent que la symétrie de dimensions n'existe pas entre les dents. La symétrie absolue des deux incisives centrales n'existe que dans $14 \%$ des cas, la différence entre les deux dents n'excède cependant pas $0,3 \mathrm{~mm}$. L'incisive latérale maxillaire est celle qui présente le plus d'asymétrie de dimension, de forme,
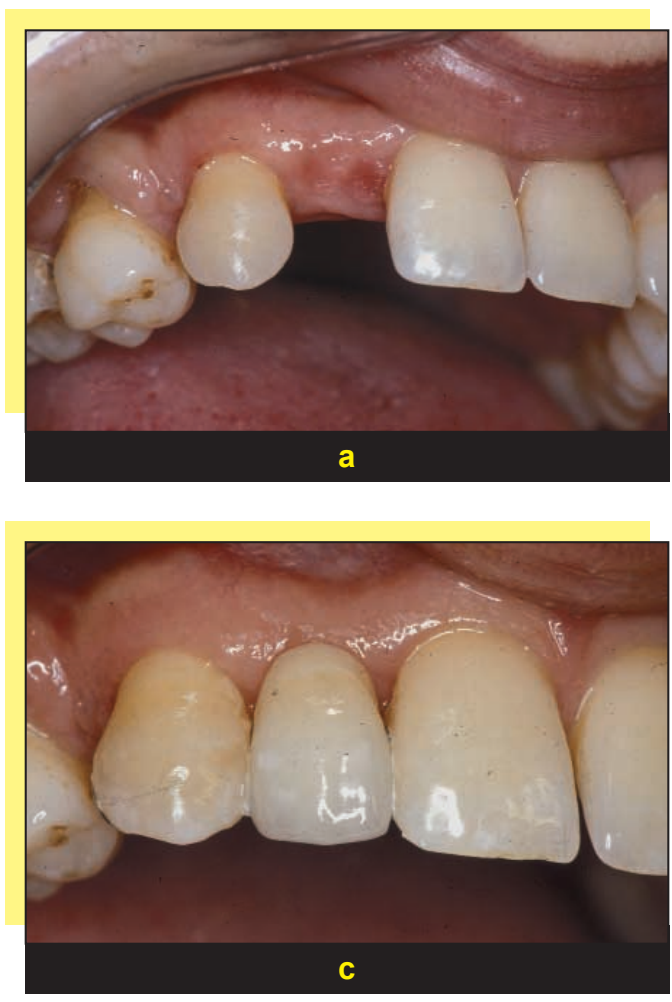

voire de présence suite à la fréquence des agénésies de cette dent (fig. 11 a à c).

Symétrie des positions: les symétries horizontales et radiantes parfaites n'existent pas. De très légères rotations, gressions, perturbent cet agencement idéal mais l'harmonie doit demeurer. C'est prothétiquement, l'élément le plus délicat à reproduire : en effet comment recréer une symétrie asymétrique ou une asymétrie symétrique.

Symétrie gingivale : la forme des limites gingivales est en relation directe avec la position des dents, il est donc naturel que la symétrie gingivale n'existe pas, ne demeurant que relative.

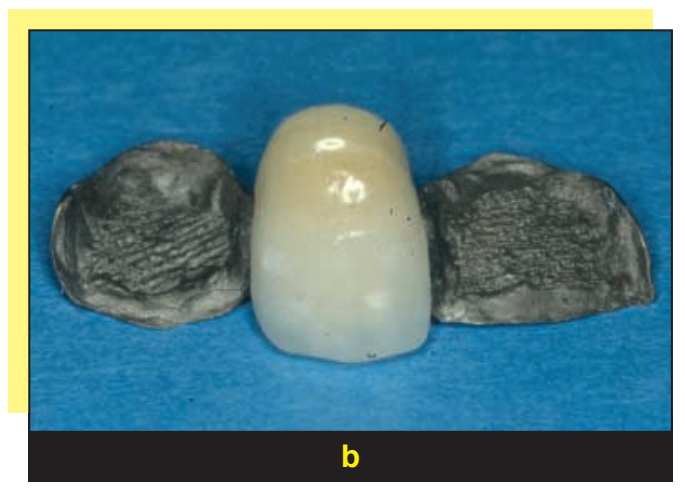

fig. 11 a à $\mathrm{c}$

a : agénésie d'une incisive latérale ;

b : aspect de l'armature du bridge collé ;

c : résultat final. 


\section{INDIVIDUALISATION}

\section{PAR LES FORMES}

La forme des dents naturelles est extrêmement riche. Elle résulte de l'accumulation de convexités, de concavités qui se succèdent, se mélangent, de lignes de force verticale, oblique, horizontale, autant de structures qui modifient imperceptiblement les formes des dents. Il incombe au laboratoire et au
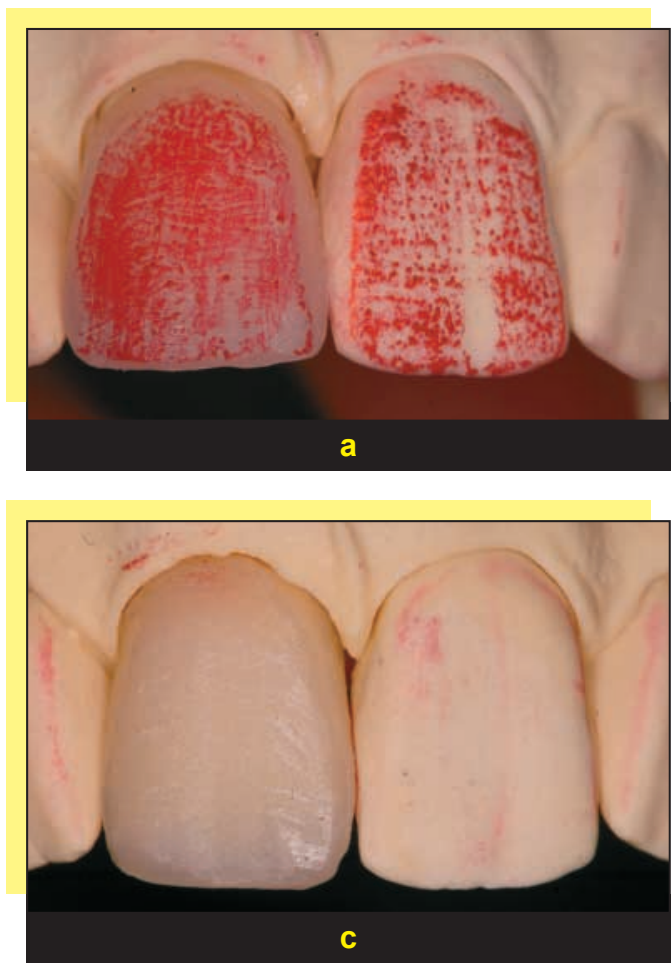

praticien de recréer ces données pour individualiser.

\section{INDIVIDUALISATION \\ PAR LA TEINTE}

En prothèse conjointe les matériaux céramiques, composites offrent des possibilités de reproduction des colorations remarquables des dents naturelles. A

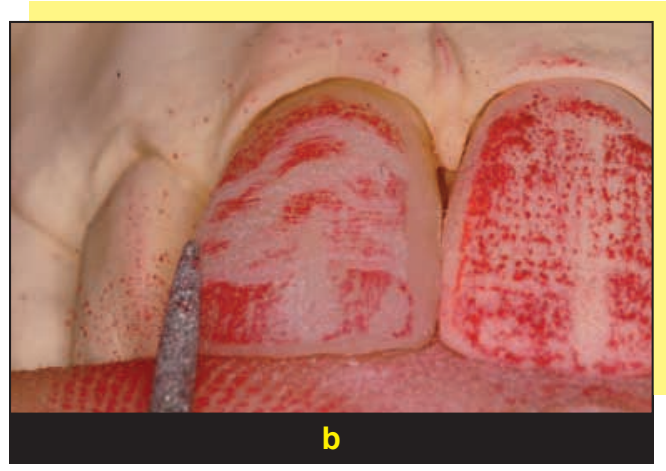

\section{fig. $12 \mathrm{a}$ à $\mathrm{c}$}

a : mise en évidence des micro reliefs sur la face vestibulaire des dents antérieures ;

b : modification de la face vestibulaire à l'aide d'une fraise diamantée ;

c : résultat final.

fig. 13 prothèse complète : aspect des dents antérieures maquillées.

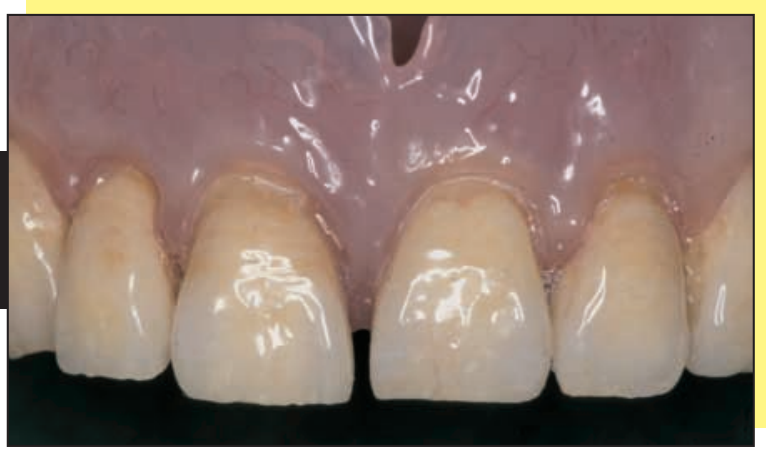


cela s'ajoutent les possibilités offertes au laboratoire ou au praticien de reproduire sur les couronnes céramique ou céramométallique les macro ou micro reliefs mineurs et majeurs (fig. 12 a à c).
Ces différentes techniques sont complétées par les possibilités de maquillage des dents prothétiques de prothèse adjointe (fig. 13)[16-17].

\section{conclusion}

La restauration du sourire est pour les patients une nécessité physique et psychologique. Cela implique que les praticiens et le laboratoire tiennent compte et appliquent les différentes lois qui régissent l'esthétique telles qu'elles ont été décrites depuis nombre de siècles.
Mais l'application pure et dure de ces règles conduira souvent à des sourires stéréotypés, uniformes, froids. Il conviendra toujours d'ajouter une petite touche personnelle pour casser cette parfaite organisation car «l'ennui naquit un jour de l'uniformité».

\section{bibliographie}

1. De Barthillat $C$. Le livre du sourire : sourire des dieux, sourire des hommes. Éditions du rocher, Paris 1992.

2. Ermiane $\mathrm{C}$, Baudet $\mathrm{BL}$, Gregori P.

Visage

et contacts humains. Paris Editions Sociales Françaises, 1969.

3. Berteretche MV. Visage esthétique, symétrie 1re partie. Cah Prothèse 1996: 93:16-24.

4. Berteretche MV, Hue O Visage esthétique, symétrie $2^{\mathrm{e}}$ partie. Cah Prothèse 1997: 98:46-57
5. Aschlein KW, Dale BG. Esthetic in dentistry a clinical approach to techniques and materials. Mosby, St louis, 2001.

6. Ricketts R. Cephalometric analysis and synthesis. Angle Orthod 1961;31: 141-156.

7. Fradeani M. Analyse esthétique : une approche systématique du traitement prothétique.

Quintessence international Paris 2007.

8. Vig RG, Brundo GC. The kinetics of anterior display.
J Prosthet Dent 1972;39: 502-506.

9. Tjan HAL, Miller GD. Some esthetic factors in smile.

J Prosthet Dent 1984;51: 24-28.

10. Rufenacht $C R$.

Fundementals of esthetics. Chicago ; Quintessence, 1990.

11. Levin El. Dental esthetic and golden. J Prosthet Dent 1978;40: 244-252.

12. Preston JD. The golden proportion revisited.

J Esthet dent 1993;5: 247-251. 
13. Brisman AS.

Esthetic: a comparaison of dentists' and patients' concept. J Am Dent Assoc 1980; 100:345-352.

14. Chiche GJ, Pinault A. Esthetics of anterior fixed prosthodontic. Chicago, Quintessence 1994.
15. Goldstein RE.

\section{Esthetic in dentistry.}

Philadelphia: Lippincott: 1976.

16. MV Berteretche, JY Ciers, O Hüe.

«Vieillissement des dents naturelles, et maquillage des dents en prothèse amovible.

1) Les dents résine.»
Synergie Prothétique 2000;2(4):271-283

17. O. Hüe, C. Nardari, MV Berteretche. «Vieillissement des dents naturelles, et maquillage des dents en prothèse amovible. 2) Les dents en porcelaine.." Synergie Prothétique 2000;2(5):327-338

\section{SUMMARY}

\section{The smile is prosthodontics or the smile praise}

\section{O. HUE}

The smile is a natural reflex, but we must add to it these numerous functions as expression of the face, feelings, attraction. So, this natural reflex is difficult to analyse, understand and reproduce. Then, the components of smile must be analyzed according to three frames: facial, labial, dental.

keywords: smile, occlusal plane, hue, color, aesthetic.
Their difficult reproductions have as goals the individualization of the teeth: individualization with position, form, hue, color. The new prosthodontic technique and protocols allow to answer to these purposes. 